\title{
Disruption of desmin-mitochondrial architecture in patients with regurgitant mitral valves and preserved ventricular function
}

\author{
Mustafa I. Ahmed, MD, ${ }^{a}$ Jason L. Guichard, MD, PhD, ${ }^{a}$ Namakkal S. Rajasekaran, PhD, ${ }^{b}$ \\ Shama Ahmad, PhD,${ }^{\mathrm{c}}$ Nithya Mariappan, PhD,${ }^{\mathrm{c}}$ Silvio Litovsky, MD, ${ }^{\mathrm{b}}$ Himanshu Gupta, MD, ${ }^{\mathrm{a}, \mathrm{d}}$ \\ Steven G. Lloyd, MD, ${ }^{\mathrm{a}, \mathrm{d}}$ Thomas S. Denney, PhD, ${ }^{\mathrm{e}}$ Pamela Cox Powell, MS, ${ }^{\mathrm{d}}$ Inmaculada Aban, PhD, ${ }^{\mathrm{f}}$ \\ James F. Collawn, PhD, ${ }^{\mathrm{g}}$ James E. Davies, MD, ${ }^{\mathrm{d}, \mathrm{h}}$ David C. McGiffin, MD, ${ }^{\mathrm{i}}$ and Louis J. Dell'Italia, MD ${ }^{\mathrm{a}, \mathrm{d}}$
}

\begin{abstract}
Objective: Recent studies have demonstrated improved outcomes in patients receiving early surgery for degenerative mitral regurgitation (MR) rather than adhering to conventional guidelines for surgical intervention. However, studies providing a mechanistic basis for these findings are limited.

Methods: Left ventricular (LV) myocardium from 22 patients undergoing mitral valve repair for American Heart Association class I indications was evaluated for desmin, the voltage-dependent anion channel, $\alpha$-B-crystallin, and $\alpha, \beta$-unsaturated aldehyde 4-hydroxynonenal by fluorescence microscopy. The same was evaluated in 6 normal control LV autopsy specimens. Cardiomyocyte ultrastructure was examined by transmission electron microscopy. Magnetic resonance imaging with tissue tagging was performed in 55 normal subjects and 22 MR patients before and 6 months after mitral valve repair.
\end{abstract}

Results: LV end-diastolic volume was 1.5 -fold $(P<.0001)$ higher and LV mass-tovolume ratio was lower in MR $(P=.004)$ hearts versus normal hearts and showed improvement 6 months after mitral valve surgery. However, LV ejection fraction decreased from $65 \% \pm 7 \%$ to $52 \% \pm 9 \%(P<.0001)$ and LV circumferential $(P<.0001)$ and longitudinal strain decreased significantly below normal values $(P=.002)$ after surgery. Hearts with MR had a $53 \%$ decrease in desmin $(P<.0001)$ and a 2.6 -fold increase in desmin aggregates $(P<.0001)$ versus normal, along with substantial, intense perinuclear staining of $\alpha, \beta$-unsaturated aldehyde 4-hydroxynonenal in areas of mitochondrial breakdown and clustering. Transmission electron microscopy demonstrated numerous electron-dense deposits, myofibrillar loss, Z-disc abnormalities, and extensive granulofilamentous debris identified as desmin-positive by immunogold transmission electron microscopy.

Conclusions: Despite well-preserved preoperative LV ejection fraction, severe oxidative stress and disruption of cardiomyocyte desmin-mitochondrial sarcomeric architecture may explain postoperative LV functional decline and further supports the move toward earlier surgical intervention. (J Thorac Cardiovasc Surg 2016;152:1059-70)

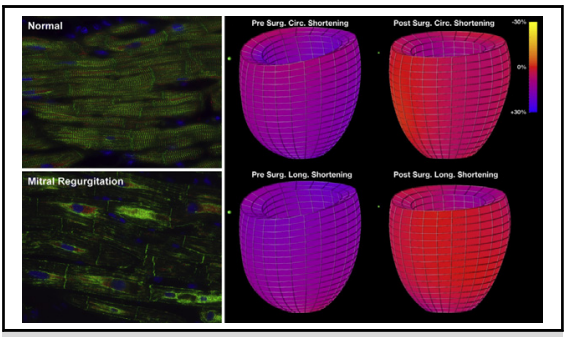

Disruption of the normal desmin pattern in MR may underpin a decrease in LV function after surgery.

\section{Central Message}

Chronic cardiomyocyte oxidative stress and disruption of the desmin cytoskeleton may explain postoperative LV functional decline in patients with well-preserved LV function and adherence to conventional guidelines.

\section{Perspective}

Recent studies report improved outcomes in patients undergoing early surgery for degenerative MR. Severe cardiomyocyte oxidative stress and desmin and mitochondrial disruption in patients with isolated MR and LV ejection fraction $>60 \%$ may underpin the decrement in LV systolic function after valve repair and further supports the move toward earlier surgical intervention.

See Editorial Commentary page 1071.
From the ${ }^{\mathrm{a}}$ Division of Cardiovascular Disease, Department of Medicine, Departments of ${ }^{b}$ Pathology; ${ }^{c}$ Anesthesiology and Perioperative Medicine; ${ }^{\mathrm{f}}$ Biostatistics; and ${ }^{\mathrm{g}}$ Cell, Developmental, and Integrative Biology, and ${ }^{\mathrm{h}}$ Division of Thoracic and Cardiovascular Surgery, Department of Surgery, University of Alabama at Birmingham, Birmingham, Ala; ${ }^{\mathrm{d} D e p a r t m e n t}$ of Veterans Affairs Medical Center, Birmingham, Ala; ${ }^{e}$ MRI Research Center, Auburn University, Auburn, Ala; and ${ }^{\mathrm{i} T h e}$ Alfred Hospital and Monash University, Melbourne, Australia. M.I.A. and J.L.G. contributed equally to this work.

This work was supported by the National Heart, Lung, and Blood Institute and the Specialized Centers of Clinically Oriented Research (grant No. P50HL077100 to L.J.D.) in cardiac dysfunction; by a Department of Veterans Affairs Merit Review
Award (grant No. 1CX000993-01 to L.J.D.); by National Institutes of Health gran No. P01 HL051952 (to L.J.D.); and National Heart, Lung, and Blood Institute Training Grant Nos. 5-T32-HL-072757 (to J.L.G) and R01HL118067 (to R.N.S.). Received for publication Jan 15, 2016; revisions received June 2, 2016; accepted for publication June 10, 2016; available ahead of print July 25, 2016.

Address for reprints: Louis J. Dell'Italia, MD, Birmingham VA Medical Center, $700 \mathrm{~S}$ 19th St, Birmingham, AL 35233 (E-mail: louis.dellitalia@va.gov). 0022-5223/\$0.00

Published by Elsevier Inc. on behalf of The American Association for Thoracic Surgery

http://dx.doi.org/10.1016/j.jtcvs.2016.06.017 

Abbreviations and Acronyms
4-HNE = 4-hydroxynonenal
BSA = bovine serum albumin
$\mathrm{LV}=$ left ventricular
LVED = left ventricular end-diastolic
LVEF = left ventricular ejection fraction
LVES = left ventricular end-systolic
MR = mitral regurgitation
MRI = magnetic resonance imaging
PBS = phosphate buffered saline
TEM $=$ transmission electron microscopy
VDAC $=$ voltage-dependent anion channel

\section{Scanning this QR code will take you to the supplemental figures and tables for this article.}

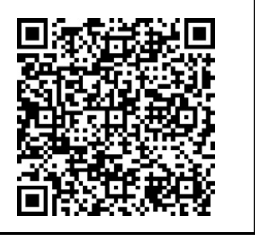

Isolated/primary mitral regurgitation (MR) is a very common heart valve disease in the United States. An estimated 2 to 2.5 million people in the United States are affected by moderate-to-severe mitral valve disease characterized by a myxomatous degeneration of the mitral valve and progressive dilating of the left ventricular (LV) chamber that leads to heart failure. With population growth and aging this number is expected to double by $2030 .{ }^{1}$ Studies in asymptomatic patients with moderateto-severe MR with LV ejection fraction (LVEF) $>60 \%$ and LV end-systolic (LVES) dimension $<40 \mathrm{~mm}$ have shown that $30 \%$ develop symptoms of heart failure or LV dysfunction over 2 years, with the majority progressing to these end points and the need for surgery over 8 years. $^{2-4}$

Because there is no effective medical therapy for asymptomatic patients with isolated MR, the latest American College of Cardiology/American Heart Association guidelines recommend surgery as a class I indication for moderate-to-severe MR in patients who are symptomatic or have progressive LV dysfunction defined by LVEF $<60 \%$ or LVES dimension $\geq 4.0 \mathrm{~cm} .{ }^{5}$ In the absence of class I indications, asymptomatic patients with LVEF $>60 \%$ and LVES diameter $<40 \mathrm{~mm}$ are only offered early repair as a class IIa recommendation in patients for whom the likelihood of a successful and durable repair without residual MR is $>95 \%$ with an expected mortality rate of $<1 \%$ when performed at a certified American Heart Association Cardiac Center of Excellence. The discretionary aspect of this recommendation is now being questioned, based on recent studies that report improved survival and decreased heart failure in patients undergoing early surgery before reaching class I criteria. ${ }^{6-8}$

The majority of guidelines pertaining to timing of surgery in MR were determined in previous studies of postoperative survival, but with less assessment of postoperative LV function. In addition, LV systolic dysfunction assessed by echocardiography is often underestimated in patients with severe MR due to an increase in preload combined with ejection into the lowpressure left atrium. Thus, it is not surprising that adhering to general guidelines has uncovered a subset of patients who do not have optimal outcomes using the triggers of end-systolic dimension and LVEF. In a recent study from Quintana and colleagues, ${ }^{8}$ there was early postoperative LV impairment in $20 \%$ of a homogeneous population of 1705 patients with severe degenerative MR and LVEF $>60 \%$. As a result of this and other similar analyses, there is now a mounting objective call for earlier surgical intervention to provide not only better postoperative survival, but also to maintain LV function.

In support of a move toward earlier surgery, we previously demonstrated increased cardiomyocyte oxidative stress and myofibrillar degeneration in patients who have moderate-to-severe MR and LVEF $>60 \%$ that decreases significantly after mitral valve repair. ${ }^{9}$ The purpose of the current investigation is to report further cardiomyocyte ultrastructural damage in patients with well-preserved LV systolic function. Recent studies in our laboratory and others have demonstrated severe disruption and breakdown of desmin in an animal model of primary volume overload and in patients with dilated heart failure, similar to the desmin changes reported in genetic defects in the DES gene. Here, we test the hypothesis that patients with isolated MR and well-preserved LV systolic function have extensive breakdown of the major intermediate filament desmin in addition to mitochondrial damage and disarray in the sarcomere units. The results of this study may explain a potential mechanism for the decline in LV function after mitral valve repair and provide a cogent foundation for earlier surgical intervention.

\section{METHODS}

\section{Study Subjects}

The study protocol was approved by the University of Alabama at Birmingham Institutional Review Board and informed consent was obtained from all MR patients and control subjects. The study was divided into 2 parts: the clinical comparison of the study group (22 MR patients) with a control group (55 volunteers) and the immunohistochemical comparison (22 specimens from MR patients) with LV specimens from 6 autopsies. Twenty-two patients (mean age, $57 \pm 12$ years; range, $34-80$ years) with severe isolated MR secondary to degenerative mitral valve disease were referred to our institution for corrective mitral valve surgery. Severe MR was documented on echocardiogram/Doppler studies and cine magnetic resonance imaging (MRI) in all cases. All patients underwent cardiac catheterization before surgery. Patients with obstructive coronary artery disease ( $>50 \%$ stenosis), aortic valve disease, or concomitant mitral stenosis were excluded. This patient population was selected from a consecutive series of patients recruited using funds from our National Institutes of Health Specialized Center for Clinically Oriented Research grant (No. P50HL077100) between June 2005 and July $2010(\mathrm{~N}=60)$, with surgery 
performed by a member of the author team (DCM). Of this population, 22 patients were selected based on the availability of remaining biopsy sample for desmin analysis and the presence of more than 3 high-powered fields of longitudinally directed cardiomyocytes for adequate desmin analysis and quantification. All patients had degenerative mitral valve disease documented by echocardiography manifested by thickening and prolapse of the mitral valve and by the surgeon's description at operation. All patients had Carpentier's functional classification type II mitral valve disease.

Patients underwent MRI with tissue tagging before and 6 months after mitral valve repair. All MRI studies were obtained within 1 month of surgery and 6 months after surgery. Only 3 patients did not have a 6-month image. MRI was also performed in 55 control volunteers (age, $45 \pm 14$ years; median, 45 years; and range, 20-70 years). These subjects were recruited for the study and were included if they did not have a history of cardiovascular disease and smoking, and were not taking any type of cardiovascular medication, including statins. All control subjects signed an informed consent form approved by the University of Alabama at Birmingham Institutional Review Board.

\section{MRI}

MRI was performed on a 1.5-T scanner (Signa GE, Milwaukee, Wis) optimized for cardiac application and LV volumes were computed from summated serial short-axis slices as previously described. ${ }^{2,9,10}$ Tagged MRI images were acquired on the same scanner with repetition/echo times of $8 / 4.4 \mathrm{~ms}$ and tag spacing of $7 \mathrm{~mm}$. Three-dimensional $\mathrm{LV}$ strain was measured from tagged images at end-systole, which was defined by visual inspection of the image data as the time frame with maximum contraction. Strain computations were conducted using an in-house software package, as previously described. ${ }^{9}{ }^{10}$ Strains were computed at midwall segments as defined by Cerqueira and colleagues. ${ }^{11}$

\section{Surgical Methods}

At surgery, $L V$ myocardial tissue was taken from the endocardium of the LV lateral posterior wall at the level of the tips of the papillary muscles in all patients. The biopsy was taken as soon as the left atrium was opened, which was immediately after cardioplegic arrest (in all cases no longer than 5 minutes after cardiopulmonary arrest). Mitral valve repair was performed through median sternotomy and standard hypothermic cardiopulmonary bypass and cold blood cardioplegia. A variety of methods were used to repair the mitral valve, including leaflet resection, chordal replacement, or a combination of those. All patients had implantation of a flexible annuloplasty band. Repair was assessed by intraoperative transesophageal echocardiography.

\section{Immunohistochemistry}

Control LV myocardial specimens were obtained at time of autopsy in patients with no evidence of myocardial or valvular disease. The specimens were obtained from a 31-year-old man who died from drug abuse, a 45-yearold man who died in a motor vehicle accident, a 24-year-old man who died from drug abuse, a 28-year-old man who died from drug abuse, a 34-yearold man with schizophrenia, and 47-year-old woman with chest pain for a week. Patient biopsies were fixed, paraffin-embedded, and processed for immunohistochemistry as previously described. ${ }^{9}$ Sections were incubated with antibodies: voltage-dependent anion channel (VDAC) (Abcam \#ab14734; 1:500 [Cambridge, Mass]), $\alpha$-B-crystallin (Enzo \#ADI-SPA223; 1:100 [Farmingdale, NY]), desmin (Abcam \#ab15200 or \#ab6322; 1:200), $\alpha, \beta$-unsaturated aldehyde 4-hydroxynonenal (4-HNE) (Millipore \#393207; 1:200 [Billerica, Mass]), and catalase (Millipore \#219010; 1:200). Alexa Fluor 488- and 594-conjugated secondary antibodies (1:700; Life Technologies, Carlsbad, Calif) with appropriate host combinations were used to stain for visualization. Nuclei were labeled with 4',6-diamidino-2-phenylindole stain $(1.5 \mu \mathrm{g} / \mathrm{mL}$, Vector Laboratories \#H-1500 [Burlingame, Calif]). Image acquisition for desmin and 4-HNE were performed on a Leica DM6000 epifluorescence microscope with Simple-PCI Imaging software (Compix, Inc). Pixel intensity was analyzed by measuring the intensity (ie, mean red and mean green) of the fields.

\section{Transmission Electron Microscopy (TEM) of LV Tissue}

Eight of 22 patients underwent TEM analysis. Patient biopsies were fixed in $2.5 \%$ glutaraldehyde/Sorensen's phosphate buffer (Electron Microscopy Sciences \#15980; Hatfield, Pa) overnight at $4^{\circ} \mathrm{C}$, as previously described by our laboratory. ${ }^{9}$ Sections were viewed using a Philips 201 TEM scanner (FEI Co, Hillsboro, Ore) by technicians at EMLabs Inc (Birmingham, Ala). Immunoelectron microscopy was used to detect desmin at the ultrastructural level, as described by Wang and colleagues. ${ }^{12}$ The human heart biopsy pieces were submerged in $0.1 \%$ glutaraldehyde $/ 2 \%$ paraformaldehyde in cardioplegic buffer $(5 \%$ dextrose, $30 \mathrm{mmol} / \mathrm{L}$ potassium chloride in phosphate buffered saline [PBS]), further fixed in the same fixative in cacodylate buffer, incubated in $0.1 \mathrm{~mol} / \mathrm{L}$ glycine/PBS, dehydrated in series of $\mathrm{N}, \mathrm{N}$ dimethyl formamide, and embedded in LR White resin. Ultrathin $(90 \mathrm{~nm})$ sections $^{10-12}$ were picked up on nickel grids $(3 \mathrm{~mm})$ and dried. Before immunolabeling, grids were rinsed in PBS and blocked for 1.5 hours with $1 \%$ bovine serum albumin (BSA), $0.1 \%$ cold-water fish skin gelatin, and $1 \%$ polysorbate 20 in PBS. Grids were then incubated in desmin antibody (Abcam \#ab15200), diluted 1:25 with 1\% BSA/PBS first for 2 hours at room temperature and then overnight at $4{ }^{\circ} \mathrm{C}$. The grids were rinsed with PBS, blocked, and incubated (at room temperature for 2 hours) with goat antirabbit immunoglobulin G tagged with colloidal gold (10-nm particle size, diluted 1:50 with 1\% BSA/PBS) (Aurion/Electron Microscopy Sciences). Sections were postfixed and counterstained with uranyl acetate and viewed on a FEI-Tecnai T12 Spirit 20 electron microscope at $120 \mathrm{kv}$.

\section{Statistical Analysis}

Data are presented as mean \pm standard deviation or counts (percent). Age and gender were compared between 22 MR patients and 55 normal controls using nonpaired $t$ test and $\chi^{2}$ test, respectively. Intensity measurements from normal and MR LVs for desmin and 4-HNE were compared with an exact Wilcoxon 2-sample test. To enable pairwise comparisons of the 3 groups of interest (normal subjects, baseline MR, and 6-month post-MV repair) in 1 model, we defined a group variable with these 3 categories. In modeling the clinical characteristics (ie, body surface area, heart rate, systolic blood pressure, and diastolic blood pressure) and MRI functional data (ie, LV end-diastolic [LVED] diameter, LVES diameter, LVED volume, LVES volume, LVEF, LVED mass-to-volume ratio, LVED sphericity index, LV circumferential strain, and longitudinal strains), we fitted a generalized linear model separately for each outcome with group as the main variable of interest using generalized estimating equations and assuming a gamma distribution. Analyses were done using the GENDMOD procedure in SAS version 9.3 software (SAS Institute, Cary, NC) using the REPEATED statement with an exchangeable working correlation structure to account for the correlation between pre- and postsurgery readings. We added age as covariate to address the imbalance between MR and normal controls in the MRI analyses. To avoid inflating the probability of a type I error due to testing these 9 primary MRI parameters, the Bonferroni-Holm stepdown method was used to adjust the significance level in the determination of the significance of the group main effects, maintaining a family-wise significance level of 0.05 . Once the group effect was deemed significant for a given MRI parameter, we performed pairwise comparisons (pre vs post, pre vs normal, and post vs normal) using a cutoff of $P<.0167$ ( 0.05 out of 3 ).

\section{RESULTS}

\section{Patient Characteristics and MRI Parameters}

Body surface area, heart rate, and blood pressure did not differ among control and pre- and postmitral valve repair groups (Table 1 and Table E1). All patients were referred to the University of Alabama at Birmingham Cardiovascular Surgery Clinic and had either tranesophageal $(\mathrm{n}=4)$ or transthoracic $(\mathrm{n}=18)$ LVES diameter $\leq 4.0 \mathrm{~cm}$ or LVEF $>55 \%$ 
TABLE 1. Demographic and clinical characteristics and magnetic resonance imaging results

\begin{tabular}{|c|c|c|c|c|}
\hline \multirow[t]{2}{*}{ Demographic characteristic } & $\operatorname{MR}(\mathbf{n}=22)$ & \multicolumn{2}{|c|}{ Normal $(\mathbf{n}=\mathbf{5 5})$} & $P$ value \\
\hline & $13(59)$ & \multicolumn{2}{|c|}{$24(43)$} & .22 \\
\hline \multicolumn{5}{|c|}{ New York Heart Association functional class } \\
\hline 1 & $6(27)$ & & & NA \\
\hline 2 & $14(64)$ & & & \\
\hline 3 & $2(9)$ & & & \\
\hline White race & $19(86)$ & & & .15 \\
\hline Age (y) & $57 \pm 12$ & & & .0006 \\
\hline Clinical characteristic & $\begin{array}{l}\text { MR presurgery } \\
\quad(\mathbf{n}=\mathbf{2 2})\end{array}$ & $\begin{array}{l}\text { 6-mo post-MVR } \\
(n=19)\end{array}$ & $\begin{array}{l}\text { Normal } \\
(\mathbf{n}=\mathbf{5 5})\end{array}$ & $\begin{array}{c}\text { Main effect } \\
P \text { value }\end{array}$ \\
\hline Body surface area $\left(\mathrm{m}^{2}\right)$ & $1.87 \pm 0.25$ & $1.84 \pm 0.27$ & $1.89 \pm 0.24$ & .2143 \\
\hline Heart rate $(\mathrm{BPM})$ & $70 \pm 11$ & $81 \pm 18$ & $71 \pm 13$ & .0724 \\
\hline Systolic blood pressure (mm Hg) & $126 \pm 18$ & $123 \pm 23$ & $119 \pm 13$ & .1609 \\
\hline Diastolic blood pressure (mm $\mathrm{Hg})$ & $74 \pm 7$ & $77 \pm 10$ & $74 \pm 10$ & .5190 \\
\hline $\operatorname{LVEDD}(\mathrm{cm})$ & $\begin{array}{c}6.01 \pm 0.65 \\
\text { vs normal } P<.0001 \\
\text { vs post-MVR } P<.0001\end{array}$ & $\begin{array}{c}5.38 \pm 0.75 \\
\text { vs normal } P=.0078\end{array}$ & $5.02 \pm 0.47$ & $<.0001$ \\
\hline $\operatorname{LVESD}(\mathrm{cm})$ & $\begin{array}{c}4.17 \pm 0.60 \\
\text { vs normal } P<.0001 \\
\text { vs post-MVR (not significant) }\end{array}$ & $\begin{array}{c}4.17(0.83) \\
\text { vs normal } P<.0001\end{array}$ & $3.52(0.41)$ & .0004 \\
\hline LVEDV (mL) & $\begin{array}{c}195(50) \\
\text { vs normal } P<.0001 \\
\text { vs post-MVR } P=.0002\end{array}$ & $\begin{array}{c}152(45) \\
\text { vs normal } P=.0012\end{array}$ & $127(27)$ & .0001 \\
\hline LVESV (mL) & $\begin{array}{c}67(20) \\
\text { vs normal } P<.0001 \\
\text { vs post-MVR (not significant) }\end{array}$ & $\begin{array}{c}74(33) \\
\text { vs normal } P<.0001\end{array}$ & $45(14)$ & .0002 \\
\hline $\operatorname{LVEF}(\%)$ & $\begin{array}{c}65(7) \\
\text { vs normal ns } \\
\text { vs post-MVR } P<.0001\end{array}$ & $\begin{array}{c}52(9) \\
\text { vs normal } P<.0001\end{array}$ & $65(6)$ & .0002 \\
\hline LV circ short $(\%)$ & $\begin{array}{c}13.4(3.2) \\
\text { vs normal } P=.0145 \\
\text { vs post-MVR } P=.001\end{array}$ & $\begin{array}{c}10.8(3.0) \\
\text { vs normal } P<.0001\end{array}$ & $15.6(1.8)$ & .0002 \\
\hline LV long short $(\%)$ & $\begin{array}{c}12.3 \pm 4.0 \\
\text { vs normal ns } \\
\text { vs post-MVR } P=.0013\end{array}$ & $\begin{array}{c}8.3 \pm 3.7 \\
\text { vs normal } P=.002\end{array}$ & $12.3 \pm 2.4$ & .0052 \\
\hline LVED sphericity & $\begin{array}{c}1.5 \pm 0.2 \\
\text { vs normal } P<.0001 \\
\text { vs post-MVR } P=.015\end{array}$ & $\begin{array}{c}1.6 \pm 0.2 \\
\text { vs normal } P=.001\end{array}$ & $1.8 \pm 0.2$ & .0005 \\
\hline LVED mass:vol & $\begin{array}{c}0.65 \pm 0.1 \\
\text { vs normal } P=.0042 \\
\text { vs post-MVR } P=.0013\end{array}$ & $\begin{array}{c}0.75 \pm 0.2 \\
\text { vs normal (not significant) }\end{array}$ & $0.75 \pm 0.2$ & .0057 \\
\hline
\end{tabular}

Values are presented as mean \pm standard deviation or count (\%). $M R$, Mitral regurgitation; $N A$, not applicable; $M V R$, mitral valve repair/replacement; $B P M$, beats per minute; $L V E D D$, left ventricular end-diastolic dimension; $L V E S D$, left ventricular end-systolic dimension; $L V E D V$, left ventricular end-diastolic volume; $L V E S V$, left ventricular end-systolic volume; $L V E F$, left ventricular ejection fraction; $L V$ circ short, left ventricular circumferential shortening; $L V$ long short, left ventricular longitudinal shortening; $L V E D$, left ventricular end diastolic.

upon visual inspection. Mean age of control subjects was $45 \pm 14$ years and of MR patients was $57 \pm 12$ years $(P=.0006)$. MR patients were New York Heart Association functional class I $(27 \%)$, class II $(64 \%)$, or class III $(9 \%)$. No MR patients had atrial fibrillation. LVED volume was increased $>50 \%$ in MR patients versus controls $(195 \pm 50 \mathrm{~mL}$ vs $127 \pm 27 \mathrm{~mL} ; P<.0001)$ and decreased after mitral valve repair $(152 \mathrm{~mL} \pm 45 \mathrm{~mL} ; P=.0002)$. LVED sphericity index (LVED length/LVED inner diameter) before and after surgery $(1.49 \pm 0.19$ and $1.58 \pm 0.19$, respectively) were significantly different from normal controls $(1.77 \pm 0.18 ; P<.0001$ and $P=.001$, respectively) but did improve postsurgery. LVED mass-to-volume ratio presurgery $(0.65 \pm 0.13 \mathrm{~g} / \mathrm{mL})$ was significantly different from normal controls $(0.75 \pm 0.17 \mathrm{~g} / \mathrm{mL} ; P=.0042)$, but did improve and did not differ from normal postsurgery $(0.75 \pm 0.20$ $\mathrm{g} / \mathrm{mL} ; P=.0013)$. Thus, there was evidence of preserved LV systolic function in the presence of adverse LV remodeling that improved after mitral valve surgery manifested by a normalization of the LVED mass-to-volume ratio. 

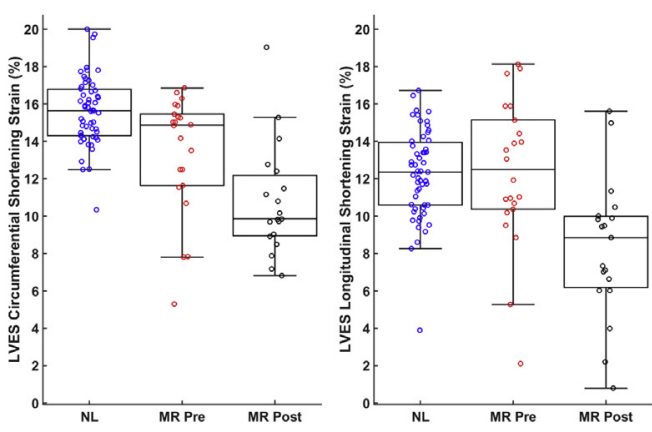

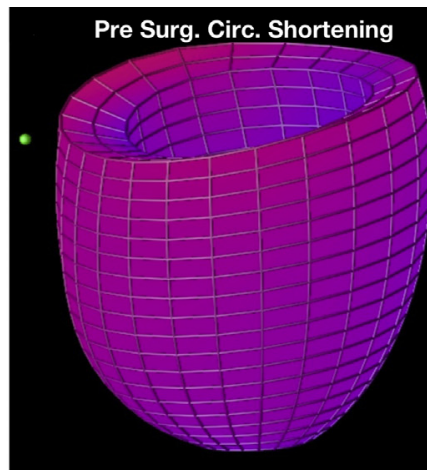

Pre Surg. Long. Shortening

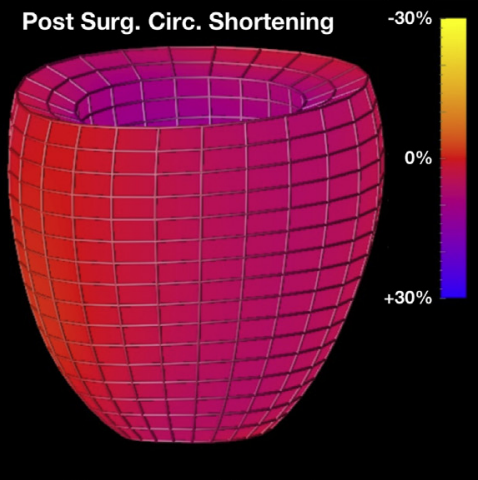

Post Surg. Long. Shortening
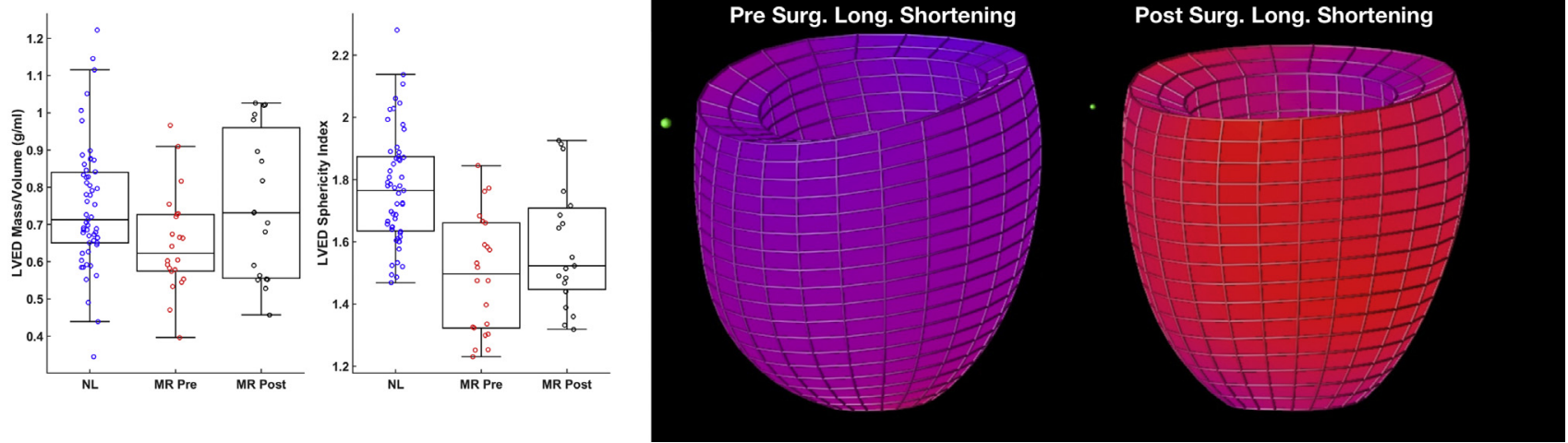

FIGURE 1. Left ventricular end-diastolic (LVED) mass-to-volume ratio, LVED sphericity index, and LV circumferential and longitudinal shortening strains in mitral regurgitation $(M R)$ patients before and 6 months after mitral valve repair, with color-coded strain maps in MR patients before and after mitral valve repair. Box-and-whisker plots demonstrate that LVED sphericity index (LVED length/LVED inner diameter) before and after surgery were significantly different from normal $(N L)$ controls $(P<.0001$ and $P=.001$, respectively), but did improve after surgery. LVED mass-to-volume ratio before surgery was significantly different from NL controls $(P=.0042)$, but did improve and did not differ from NL after surgery $(P=.0013)$. LV longitudinal systolic shortening strains were not different from NL controls before surgery, but were decreased after mitral valve repair versus before surgery $(P=.0013)$ and relative to NL control subjects $(P=.0020)$. The color-coded strain map demonstrates the significant global decrease in circumferential and longitudinal strains in a representative MR patient after mitral valve repair. $L V E S$, Left ventricular end-systolic.

LVEF decreased from $65 \% \pm 7 \%$ to $52 \% \pm 9 \%$ $(P<.0001)$ after surgery. LVES volume was higher in MR versus controls $(67 \pm 20 \mathrm{~mL}$ vs $45 \pm 14 \mathrm{~mL}$; $P<.0001)$ and did not change significantly postsurgery. LVES dimension changed in similar directions as LV volumes. LV circumferential systolic shortening strains were significantly decreased following mitral valve repair versus before surgery $(P<.001)$ and versus normal control subjects $(P<.0001)$. LV longitudinal systolic shortening strains were not different from normal controls before surgery, but were decreased following MV repair versus before surgery $(P=.0013)$ and relative to normal control subjects $(P=.0020)$ (Figure 1$)$. The color-coded strain map in Figure 1 demonstrates the significant global decrease in circumferential and longitudinal strains in a representative MR patient after mitral valve repair.

\section{Disruption of Cardiomyocyte Cytoskeletal- Mitochondrial Architecture in MR Patients}

TEM images at $4500 \times$ in 3 different representative MR patients with LVEF $>60 \%$ (Figure 2, left panels) demonstrate numerous clusters of small round mitochondria with complete disruption of the normal mitochondrial linear registry that is normally in close proximity to the sarcomeric myofibrils. In patients 1 and 2 (Figure 2), white boxes demarcate the perinuclear areas at $4500 \times$ and accompanying $16,000 \times$ images to the right demonstrate large electron-dense aggregates containing lipid droplets consistent with lipofuscin. There are numerous lipid droplets throughout the myocardium. In patient 3 (Figure 2, bottom panels), the white box in the $4500 \times$ image marks an interfibrillar area with mitochondrial breakdown and areas of granulofilamentous protein aggregates within autophagic vacuoles (patient 3, right panel, white arrow).

\section{Desmin Degradation in the Left Ventricle of Patients With Isolated MR}

A normal human left ventricle has a striated registry of desmin staining in Z-discs and more intense staining at intercalated discs (Figure 3, A, arrowheads) with VDAC staining mitochondria in a single linear registry between sarcomeres. MR hearts from 2 patients with LVEF $>60 \%$ (Figure 3, $B$ and $C$ ) demonstrate disrupted or near complete 

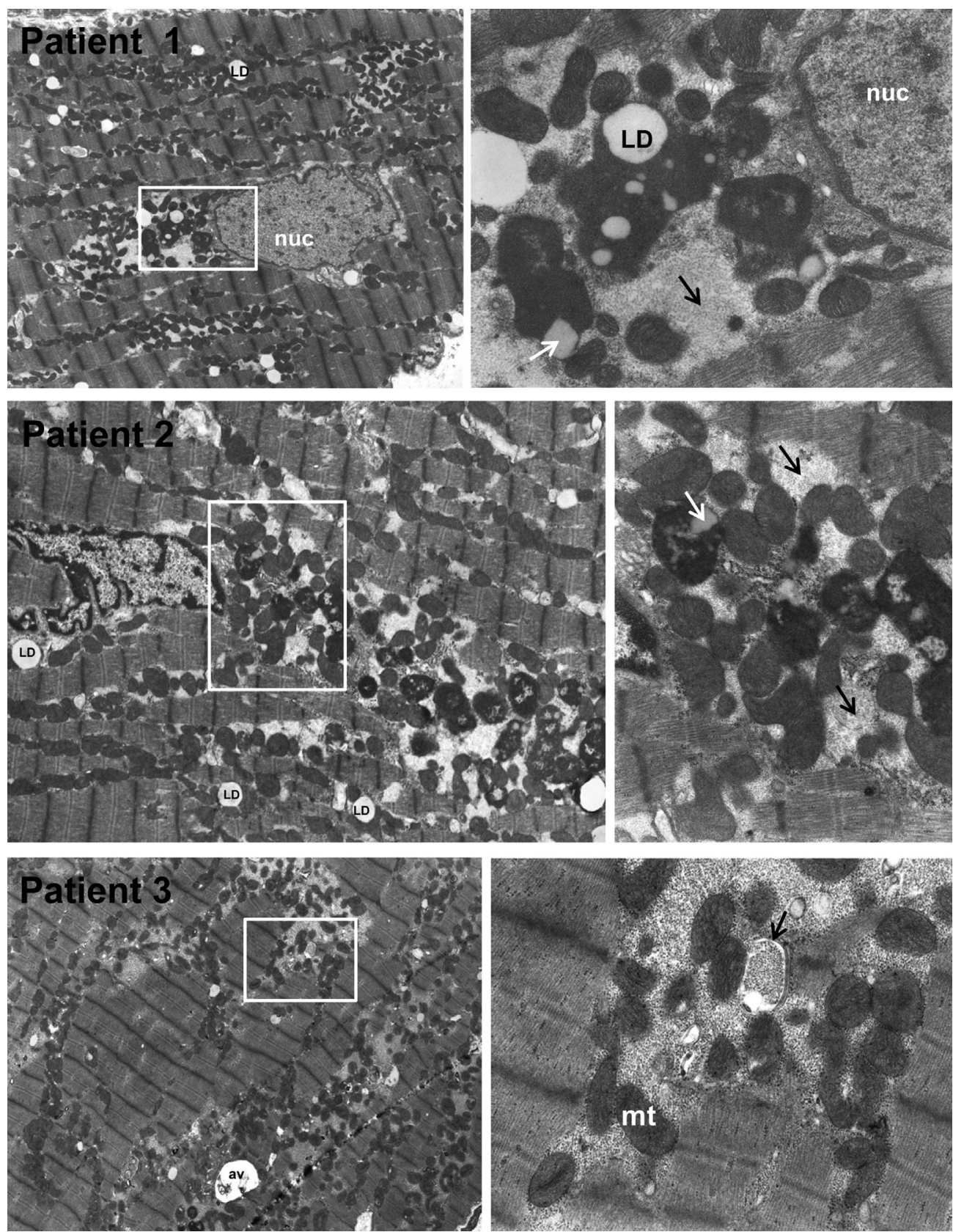

FIGURE 2. Transmission electron microscopy of the left ventricle of 3 patients with mitral regurgitation and left ventricular ejection fraction $>60 \%$. Left panels, In Patients 1 through 3, 4500× left ventricle images demonstrate extensive myofibrillar breakdown amid mitochondrial ( $m t$ ) clustering of various small sizes. Right panels $(16,000 \times$ images of the white boxes in the $4500 \times$ images), There are extensive granulofilamentous protein aggregates $($ black arrows) representing cytoskeletal and myofibrillar degeneration. Left panels, In patients 1 and 2, white boxes outline the 16,000× images. Right panels, Perinuclear areas with electron-dense bodies consistent with lipofuscin also containing lipid droplets $(L D s)$ in interfibrillar areas and in electron-dense bodies (patient 2) (white arrow). In Patient 3,16,000× image demonstrates extensive granulofilamentous protein aggregates (light gray) in areas of myofibrillar and cytoskeletal degeneration being engulfed by autophagic vacuoles (black arrow). nuc, Nucleus; $a v$, autophagic vacuoles.

absence of desmin in some cardiomyocytes. Other areas have intense desmin staining, especially in perinuclear areas, which is consistent with desmin aggregates (Figure 3, $B$ and $C$, white arrows). There is a $50 \%$ decrease in desmin staining versus normal hearts $(P<.0001)$ and a significant increase in desmin aggregates versus normal $(P<.0001)$ (Figure 3,D). There are areas of increased VDAC staining, indicating focal mitochondrial clustering, as seen in the TEM examples in Figure 2. Figure 4 demonstrates TEM with desmin immunogold labeling in the MR 

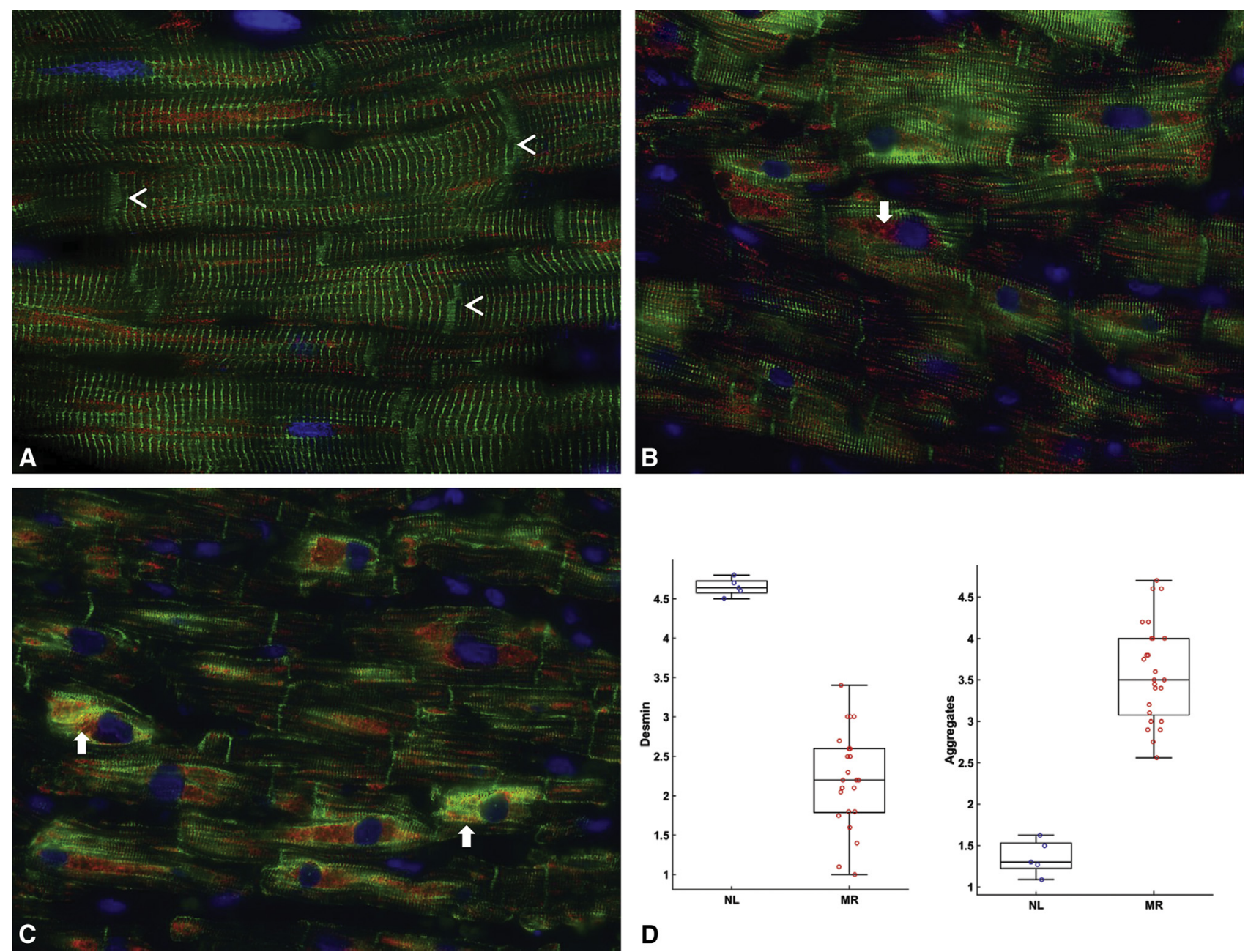

FIGURE 3. Immunohistochemistry of desmin and voltage-dependent anion channel (VDAC) in A, Normal human heart (NL) and B, Patients with mitral regurgitation $(M R)$ and left ventricular ejection fraction $>60 \%$. A, NL left ventricle demonstrates a regular pattern of desmin (green, arrowheads) along Z-lines between intercalated discs (arrowheads). Coimmunostaining with VDAC (red) shows that mitochondria are in linear register in the interfibrillar areas and grouped in perinuclear locations (blue). B and C, MR hearts demonstrate loss of desmin interspersed with areas of intense desmin staining that costains with VDAC (arrows), especially in the perinuclear areas (arrows). D, Box-and-whisker plots with the data points superimposed to show the quantitation of desmin loss and aggregate as percentage of area of cardiomyocytes. Desmin loss as percent of cardiomyocytes: Grade $1=100 \%$ loss; Grade $2=75 \%$ loss, $25 \%$ intact; Grade $3=50 \%$ loss, $50 \%$ intact; Grade $4=25 \%$ loss, $75 \%$ intact; and Grade $5=100 \%$ intact ( $\mathrm{N}=22$ patients). Desmin staining versus NL $(P<.0001)$. Desmin aggregates as percent of cardiomyocytes: Grade $1=$ no aggregates, Grade $2=25 \%$ aggregates, Grade $3=50 \%$ aggregates, Grade $4=75 \%$; and Grade $5=100 \%$ aggregates $(\mathrm{N}=22$ patients). Desmin aggregates versus NL $(P<.0001)$.

heart of a patient with LVEF of $65 \%$. There is marked disruption and degradation of the mitochondrial sarcomeric units (Figure 4, A). Boxes define areas of higher magnification (Figure 4,B) of immunogold labeling for desmin (Figure 4, black dots) in the areas of myofibrillar degradation near the interfibrillar disorganization of mitochondria.

Figure 5, $A$ - $C$, demonstrates the normal location of $\alpha$-Bcrystallin in the Z-disc (Figure 5, B) forming a striated pattern with desmin, as seen in the merged image in Figure 5, $C$. In MR, there is translocation of $\alpha$-B-crystallin to the perinuclear regions (Figure 5, $E$ and $H$ ) that costains with desmin, especially in the perinuclear area, as seen in the merged images (Figure 5, $F$ and $I$ ). This $\alpha$-B-crystallin translocation coincides with the extensive perinuclear granulomatous debris and electron-dense aggregates demonstrated in TEMs in patients 1 and 2 in Figure 2. This translocation of $\alpha$-B-crystallin from the Z-disc to the perinuclear area in the MR heart, along with costaining with desmin in merged images (Figure 5, $F$ and $I$ ), is consistent with its chaperone function of clearing desmin breakdown products. However, the extensive accumulation of both des$\min$ and $\alpha$-B-crystallin suggests a failure to clear cytoskeletal breakdown products. 


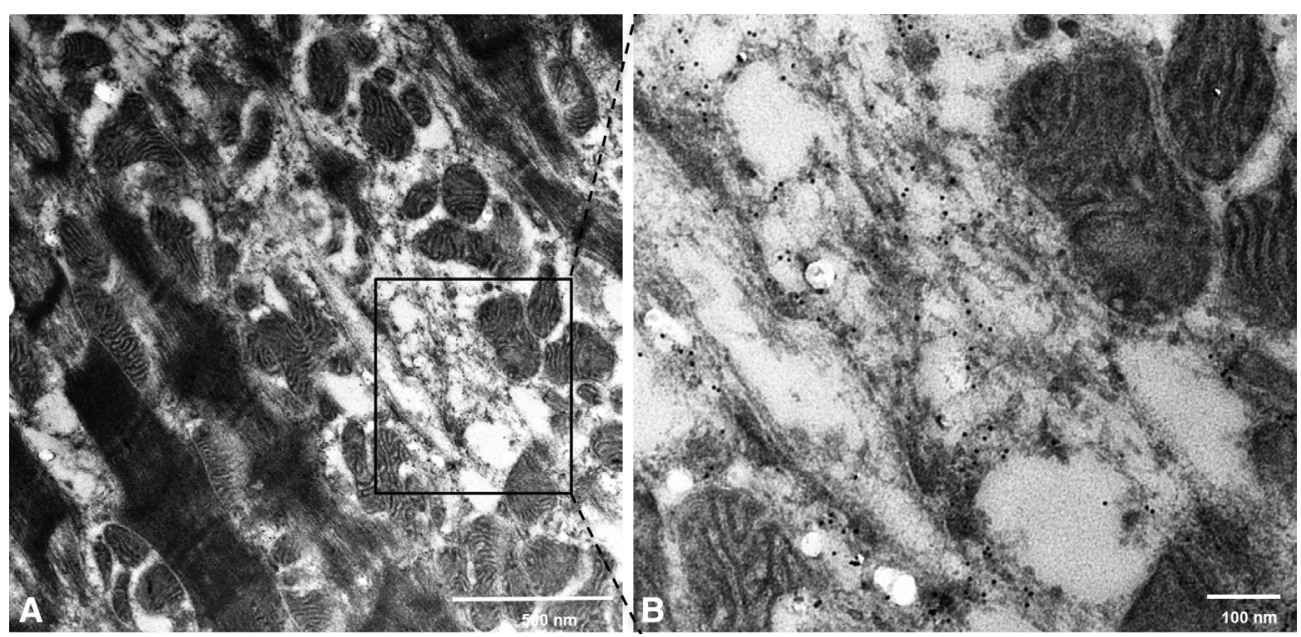

FIGURE 4. Transmission electron microscopy (TEM) immunogold labeling for desmin in a heart with mitral regurgitation. A, Lower magnification with scale bar on bottom right TEM of the heart of patient with left ventricular ejection fraction of $65 \%$ demonstrates marked disruption and degradation of the mitochondrial sarcomeric units. B, Higher magnification of the area defined by the black box in A of immunogold labeling demonstrates desmin (black dots) in areas of sarcomere/mitochondrial breakdown. Note the desmin in the areas of myofibrillar degradation near the interfibrillar disorganization of mitochondria.

\section{Evidence of Excessive Oxidative Stress in MR Cardiomyocytes}

One of the major causes of desmin disruption/aggregates and mitochondrial disarray is excessive oxidative stress. Oxidative stress is strongly indicated by $4-\mathrm{HNE}$, in particular lipid peroxidation, that is increased 2-fold $(P=.011)$ in MR hearts. Figure 6 demonstrates the significant increase in $4-\mathrm{HNE}$ in 3 patients with $\mathrm{LVEF}>60 \%$. There is a strong signal in the perinuclear region that coincides with VDAC staining in Figure 3 and in the TEM images of patients 1 and 2. The colocalization of 4-HNE and VDAC is consistent with lipid peroxidation of the membrane-rich mitochondria in cardiomyocytes. The increase in 4-HNE is associated with a moderate increase in catalase, especially in the perinuclear regions in the same MR patients in Figure $7(B$ to $D)$ compared with a normal left ventricle (Figure $6, A$ ). The increase in catalase has also been reported in heart failure patients in response to increased oxidative stress. ${ }^{13}$

To address the question of generalizability of the biopsy findings to a more contemporary patient population, we include 6 recently studied patients examined under funding of a National Institutes of Health Program Projects Grant (No. P01HL051952 - 21A1) (Table E2). These are consecutive patients referred to an author (J.E.D.) for surgical opinion regarding degenerative MR over a period of 5 months. All patients provided signed informed consent and had degenerative MR documented by echocardiography/Doppler examination and at the time of surgery. Inclusion and exclusion criteria were the same as in the main patient population and all patients had Carpentier's functional classification type II mitral valve disease and were within American Heart Association class I echocardiographic guidelines (Table E2). Representative TEMs in 2 patients demonstrate numerous electron-dense particles with mitochondrial clustering and sarcomere breakdown (Figure E1). In these same patients, there was significant degradation of desmin and accumulation of aggregates (Figure E2) $(P=.0043)$ with intense 4-HNE staining.

\section{DISCUSSION}

Our study results demonstrate breakdown of desmin and mitochondrial/sarcomeric disruption in the cardiomyocytes of patients with well-preserved LV systolic function. These findings, along with a decrease in LV function after mitral surgery, suggest that despite a significant decrease in LVED volume and normalization of LVED mass-to-volume ratio, disruption of the cardiomyocyte cytoskeletal-mitochondrial architecture may preclude an improvement in LV systolic function. Importantly, these results provide a potential mechanism for recent findings that a substantial subset of patients have unfavorable outcomes despite adherence to guideline triggers and LVEF $>60 \%$ before surgery.

In the current study, we utilized immunofluorescence microscopy, TEM, and immuno-TEM to characterize the severe desmin cytoskeletal disruption in the MR hearts. TEM findings in our MR patients are similar to cardiac findings in patients with mutations of the DES gene ${ }^{14,15}$ or its main chaperone, $\alpha$-B-crystallin, ${ }^{16}$ as well as other cytoskeletal cardiomyopathies. ${ }^{17}$ Desmin is the predominant intermediate filament protein expressed in the heart, interacts with other structural proteins at the Z-disc, and extends from the subsarcolemmal membrane to the nucleus. ${ }^{18}$ This continuous 3-dimensional cytoskeletal network maintains the close spatial relationship between the sarcomere contractile apparatus and the mitochondria and provides cellular integrity and the mechanochemical signaling 

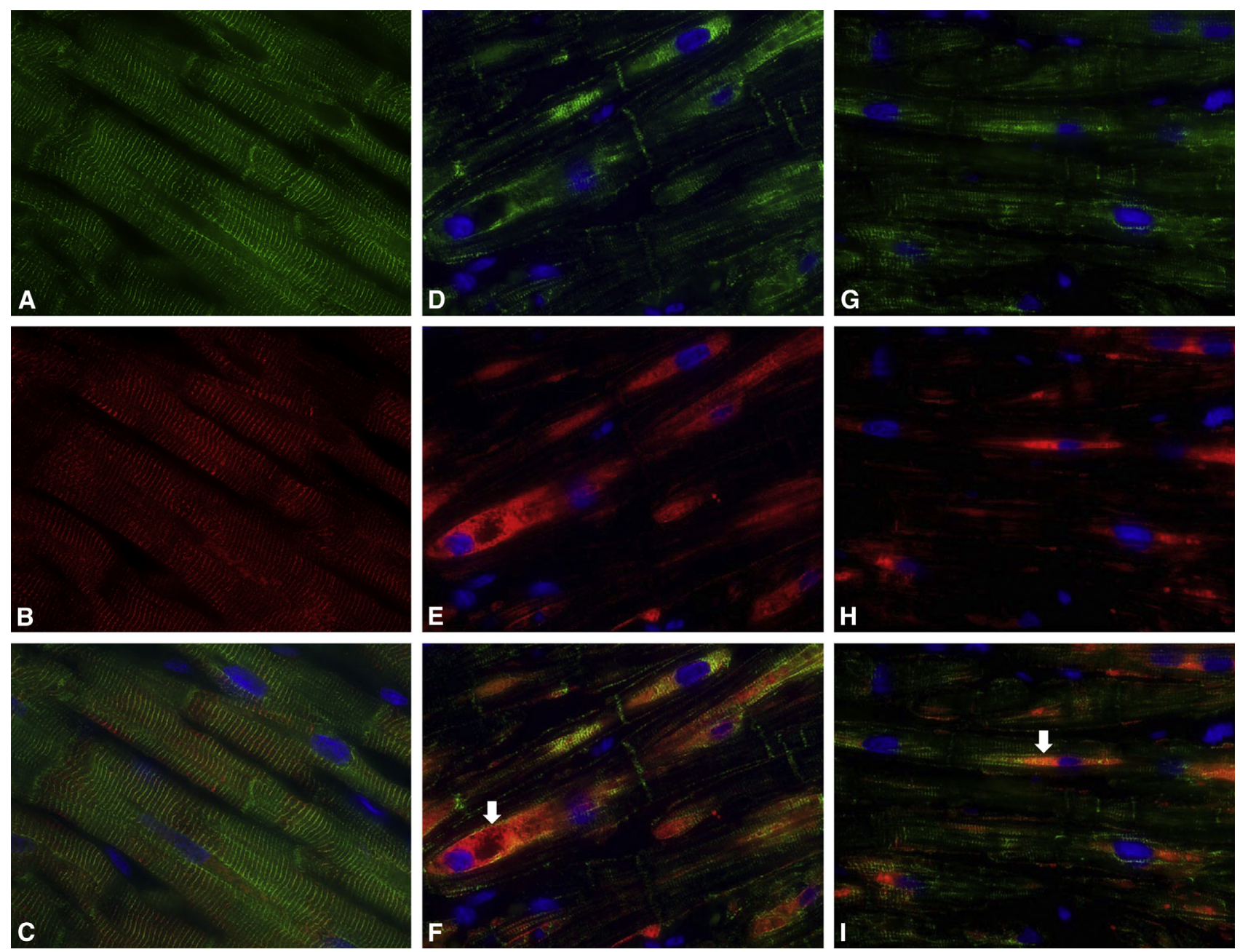

FIGURE 5. Immunohistochemistry for desmin (green) and $\alpha$-B-crystallin (red) in hearts with mitral regurgitation and normal hearts. In a normal heart, A, desmin (green) and B, $\alpha$-B-crystallin (red) are C, colocalized to the Z-disc. D-F, In hearts with mitral regurgitation in patients with left ventricular ejection fraction of $68 \%$ and G-I, Left ventricular ejection fraction of $62 \%$, there is extensive desmin loss, along with areas of aggregation most prominent in the perinuclear region (blue). $\mathrm{F}$ and I, In hearts with mitral regurgitation, $\alpha \beta$-crystallin relocates to the perinuclear region (arrows) with desmin aggregates.

necessary for sarcomere growth and force transmission. ${ }^{18}$ Currently, 49 mutations have been identified in the DES gene that alter the desmin filament assembly process ${ }^{19}$ and result in disorganization of the desmin network and accumulation of desmin-containing aggregates. Transgenic mice with a missense mutation (R120G) of $\alpha$-B-crystallin recapitulate the human cardiomyopathic phenotypes, with desmin disruption and formation of perinuclear $\alpha$-B-crystallin aggregates and progression to a dilated cardiomyopathy by age 5 to 7 months. ${ }^{20}$ Disorganization of desmin and mitochondria functional complexes with myofibrils and the sarcoplasmic reticulum are present before the onset of cardiac and skeletal muscle failure in these animal models. ${ }^{17,21}$

Although desmin disintegration is common in various forms of genetic cardiomyopathies caused by mutations in structural proteins, our findings are unique in that the MR hearts exhibiting chronic oxidative stress had desmin breakdown and the compensatory chaperone-mediated protection
( $\alpha \beta$-crystallin) failed to prevent progressive degradation and accumulation of desmin. In MR hearts, the extensive desmin breakdown and small clusters of mitochondria are accompanied by relocalization of $\alpha$-B-crystallin from the $\mathrm{Z}$-disc to the perinuclear area, where it accumulates along with electron-dense aggregates and intense 4-HNE staining. It is known that 4-HNE is a highly reactive $\alpha, \beta$-unsaturated aldehyde generated by reactive oxygen species reacting with polyunsaturated fatty acids in biological membranes, in particular the inner membrane of mitochondria. ${ }^{22}$ The intense 4-HNE staining is seen in the interfibrillar and perinuclear areas of abundant damaged mitochondria of an MR heart and its concentration in left ventricle tissue has been related to severity of LV dysfunction in patients with heart failure. ${ }^{22}$ The most abundant small heat shock protein in the heart is $\alpha$-B-crystallin, ${ }^{23}$ which serves as a chaperone for desmin and other proteins by preventing them from aggregating under stress conditions. In MR hearts, the dense 

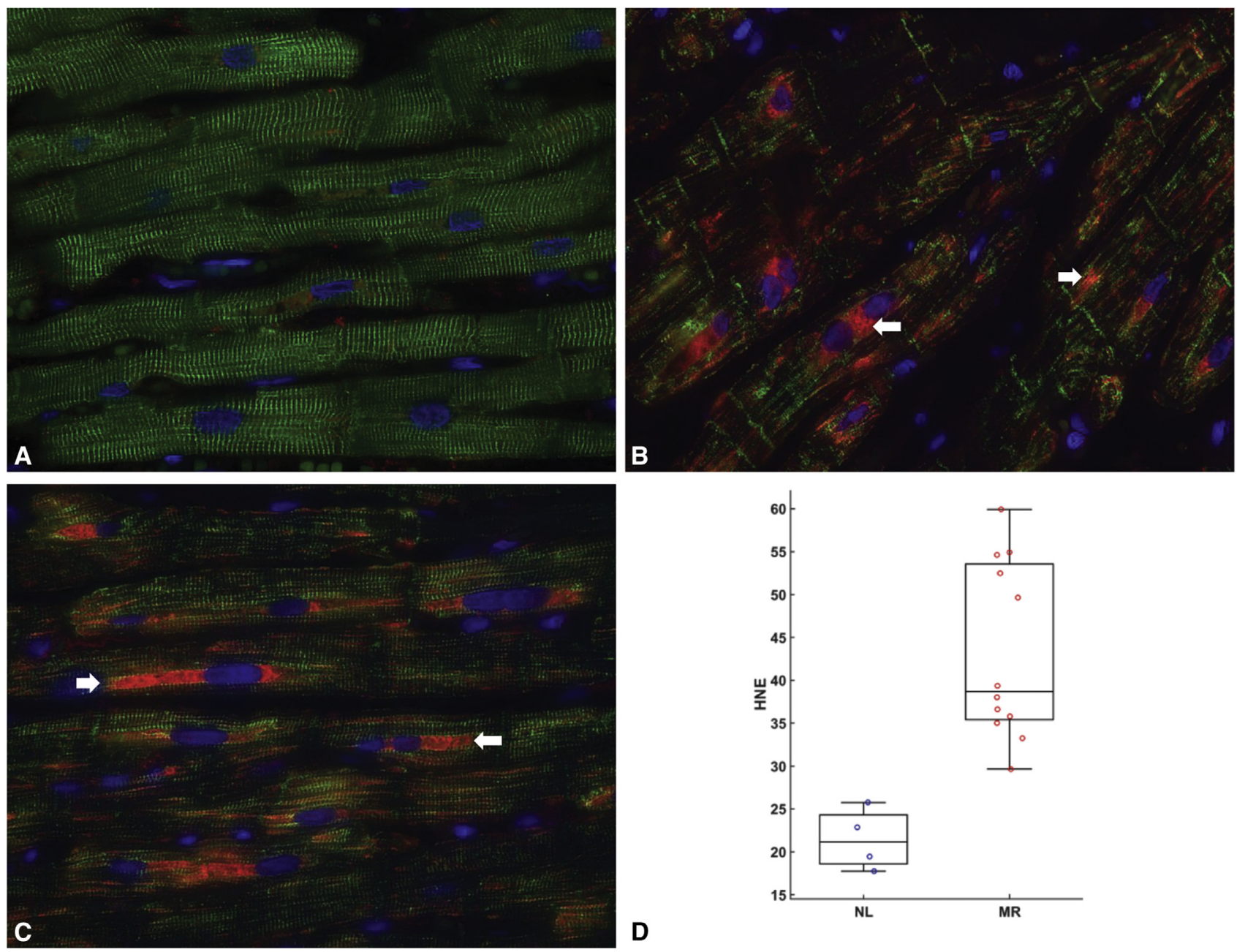

FIGURE 6. Immunohistochemistry for desmin (green) and 4-hydroxynonenal (4-HNE) (red) in normal hearts ( $N L)$ and hearts with mitral regurgitation $(M R)$. A, NL has striated pattern of desmin (green) with very little 4-HNE staining (red). B and C, In MR there is extensive desmin loss, along with 4HNE staining, especially in the perinuclear areas (arrows), that is increased 2-fold. D, Intensity of 4-HNE as percent area of cardiomyocytes. Intensity of 4-HNE in MR versus NL $(P=.012)$

perinuclear staining suggests a failure to clear desmin fragments and other oxidatively modified proteins, as suggested by the presence of lipofuscin-like electron-dense particles.

Desmin cleavage and the formation of desmin amyloidlike oligomers has recently been reported in failing human hearts and in the hearts of dogs with dyssynchronous heart failure. $^{24}$ We have reported desmin breakdown and increased cardiomyocyte oxidative stress in rats with the primary volume overload of aortocaval fistula and in isolated cardiomyocytes subjected to cyclical stretch. ${ }^{25}$ Other studies have recently shown a similar disruption and patchy loss of desmin in the left ventricles of patients with ischemic cardiomyopathy that is related to the extent of LV dysfunction and poor prognosis. ${ }^{26,27}$ The relation of desmin breakdown to long-term survival and to severity of $\mathrm{LV}$ dysfunction in human heart failure provides compelling evidence for a nongenetic cause of desmin degradation in the pathophysiology of LV functional deterioration in human heart failure and in our MR patients.

Although excessive oxidative stress is a major factor, the mechanism of desmin breakdown in the pathophysiology of MR or heart failure is multifactorial. In addition to cardiomyocyte 4-HNE, we found an increase in catalase, which is a hydrogen peroxide scavenging enzyme that is increased in response to increased oxidative stress in failing human hearts. ${ }^{13}$ These markers of increased oxidative stress are consistent with our previous report of increased xanthine oxidase in patients with isolated MR and LVEF $>60 \% .{ }^{9} \mathrm{Hu}-$ man isolated MR has also been associated with upregulation of tumor necrosis factor- $\alpha$ expression ${ }^{28}$ and increased adrenergic drive, ${ }^{29,30}$ which are related to severity of LV dilatation and systolic dysfunction. Desmin is a major substrate for the intracellular calcium dependent protease calpain, which is activated during the high intracellular free calcium levels 

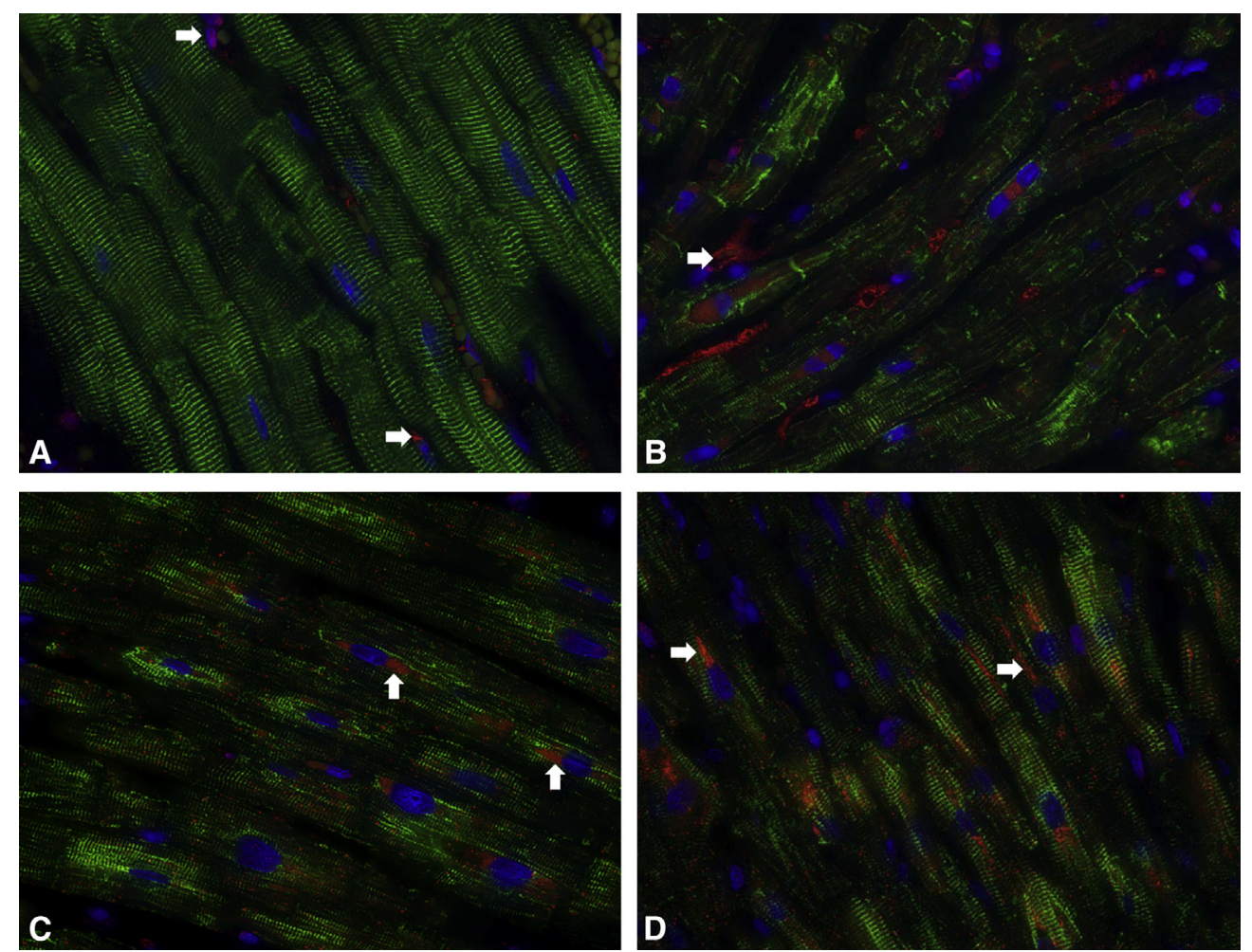

FIGURE 7. Immunohistochemistry for desmin (green) and catalase (red) in normal hearts and hearts with mitral regurgitation. A, A normal heart has the striated desmin with very little catalase staining, except in some interstitial cells (arrows). B-D, In 3 hearts with mitral regurgitation, there was extensive desmin loss along with increased catalase staining, particularly in the perinuclear regions (arrows).

achieved during ischemia/reperfusion, ${ }^{31,32}$ but also with increased tumor necrosis factor- $\alpha$ and excessive $\beta$ adrenergic signaling and oxidative stress. ${ }^{32}$ It has been shown that $\beta_{1}$-adrenergic receptor blockade improves cardiomyocyte and LV function and reverses myofibrillar degradation in dogs with isolated experimental MR, ${ }^{33,34}$ improves LV diastolic and systolic function over 2 years in patients with isolated $\mathrm{MR}^{2}$ and decreases oxidative stress and 4HNE production in the left ventricles of patients with heart failure. ${ }^{22}$ These findings support a link of incessant adrenergic drive and cardiomyocyte oxidative stress to protease activation and cytoskeletal breakdown in the adverse LV remodeling of a heart with MR.

In patients with isolated MR, the 10-unit decrease in LVEF after mitral valve surgery has in part been attributed to a LV volumetric adjustment brought about by a decrease in preload combined with correction of ejection into the low-pressure left atrium. In our MR patients, circumferential and longitudinal shortening strains decreased to below normal after mitral valve repair, despite normalization of the LVED mass-to-volume ratio, which is an index of wall stress. We previously reported persistence of decreased LV shortening strains at 1 year, ${ }^{18}$ suggesting that the decrease in LV systolic function may be due to persistent cytoskeletal-myofibrillar-mitochondrial damage. Indeed, recent studies demonstrating an increased incidence of heart failure and mortality in MR patients with class I echocardiography characteristics (LVES dimension $<4.0$ and LVEF $>60 \%$ ) also suggests more severe myocardial damage as a mechanism of this outcome. ${ }^{6-8}$

It is now well appreciated that even with strict adherence to guideline recommendations, there is a decrease in LVEF and an unacceptable incidence of heart failure after surgical repair. The severe disruption of desmin and excessive cardiomyocyte oxidative stress in our patients with wellpreserved LV systolic function now provides novel insight into the mechanisms of adverse LV spherical remodeling and a potential explanation for improved outcome with surgery before traditional guideline triggers. Considering the extensive mitochondrial disarray and the more chronic autophagic vacuoles and electron-dense aggregates, we believe this is not a result of diastolic arrest. This contention is supported by work in dogs by Kamlot and colleagues, ${ }^{35}$ demonstrating preserved mitochondrial structure and sarcomeric integrity in the left ventricle endomyocardium of dogs undergoing warm or cold cardioplegia. However, we cannot rule out the possibility of normalization of the ultrastructural derangement after repair or that the desmin changes are unrelated to the natural history of the ventricle. The major point of our current investigation is that severe ultrastructural damage is already present at the time of surgery; that is, by the time symptoms have developed or 
guidelines criteria have been achieved. Using a 3-dimensional analysis of cine-MRI in patients with degenerative MR, we demonstrated ${ }^{10}$ that LVES dimension belies the extensive LV spherical remodeling distal to the base of the heart, militating for an LVES volume rather than LVES dimension indication for surgical intervention. Further studies are needed to establish prospectively the value of LVES volume/sphericity and novel circulating biomarkers in predicting LV function after MV repair.

\section{Conflict of Interest Statement}

Authors have nothing to disclose with regard to commercial support.

\section{References}

1. Enriquez-Sarano M, Akins CW, Vahanian A. Mitral regurgitation. Lancet. 2009; 373:1382-94.

2. Ahmed MI, Aban I, Lloyd SG, Gupta H. Howard G, Inusah S, et al. A randomized controlled Phase IIb trial of Beta-1 receptor blockade in isolated degenerative mitral regurgitation. J Am Coll Cardiol. 2012;60:833-8.

3. Suri RM, Aviernos J-F, Dearani JA, Mahoney DW, Michelena HI, Schaff HV, et al Management of less-than-severe mitral regurgitation: should guidelines recommend earlier surgical intervention. Eur J Cardiovasc Surg. 2011;40:496-502.

4. Enriquez-Sarano M, Avierinos JF, Messika-Zeitoun D, Detaint D, Capps M, Nkomo V, et al. Quantitative determinants of the outcome of asymptomatic mitral regurgitation. $N$ Engl J Med. 2005;352:875-83.

5. Nishimura RA, Otto CM, Bonow RO, Carabello BA, Erwin JP III, Guyton RA, et al. 2014 AHA/ACC Guideline for the management of patients with valvular heart disease: report of the American College of Cardiology/American Heart Association Task Force on Practice Guidelines. Circulation. 2014;129:2440-92.

6. Enriquez-Sarano M, Suri RM, Clavel MA, Mantovani F, Michelena HI, Pislaru S, et al. Is there an outcome penalty linked to guideline-based indications for valvular surgery? Early and long-term analysis of patients with organic mitral regurgitation. J Thorac Cardiovasc Surg. 2015;150:50-8.

7. Goldstone AB, Patrick WL, Cohen JE, Aribeana CN, Popat R, Woo YJ. Early surgical intervention or watchful waiting for the management of asymptomatic mitral regurgitation: a systematic review and meta-analysis. Ann Cardiothorac Surg. 2015;4:220-9.

8. Quintana E, Suri RM, Thalji NM, Daly RC, Dearani JA, Burkhart HM, et al. Left ventricular dysfunction after mitral valve repair-the fallacy of "normal" preoperative myocardial function. J Thorac Cardiovasc Surg. 2014;148:2752-62.

9. Ahmed M, Gladden JD, Litovsky S, Lloyd SG, Gupta H, Inusah S, et al Myofibrillar degeneration, oxidative stress and post-surgical systolic dysfunction in patients with isolated mitral regurgitation and pre-surgical LV ejection fraction $>$ 60\%. J Am Coll Cardiol. 2010;55:671-9.

10. Schiros CG, Dell'Italia LJ, Gladden JD, Clark D III, Aban I, Gupta H, et al. Magnetic resonance imaging with three-dimensional analysis reveals important left ventricular remodeling in isolated mitral regurgitation: implications beyond dimensions. Circulation. 2012;125:233-42.

11. Cerqueira MD, Weissman NJ, Dilsizian V, Jacobs AK, Kaul S, Laskey WK, et al. Standardized myocardial segmentation and nomenclature for tomographic imaging of the heart: a statement for healthcare professionals from the Cardiac Imaging Committee of the Council on Clinical Cardiology of the American Heart Association. Circulation. 2002;105:539-42.

12. Wang X, Osinska H, Dorn GW II, Nieman M, Lorenz JN, Gerdes AM, et al. Mouse model of desmin-related cardiomyopathy. Circulation. 2001;103:2402-7.

13. Dieterich S, Bieligk U, Beulich K, Hasenfuss G, Prestle J. Gene expression of antioxidative enzymes in the human heart. Increased expression of catalase in the end-stage failing heart. Circulation. 2000;101:33-9.

14. Schröder R, Goudeau B, Simon MC, Fischer D, Eggermann T, Clemen CS, et al. On noxious desmin: functional effects of a novel heterozygous desmin insertion mutation on the extrasarcomeric desmin cytoskeleton and mitochondria. Hum Mol Genet. 2003;12:657-69.

15. Bär H, Fischer D, Goudeau B, Kley RA, Clemen CS, Vicart P, et al. Pathogenic effects of a novel heterozygous R350P desmin mutation on the assembly of desmin intermediate filaments in vivo and in vitro. Hum Mol Genet. 2005;14: 1251-60.

16. Vicart P, Caron A, Guicheney P, Li Z, Prévost MC, Faure A, et al. A missense mutation in the alphaB-crystallin chaperone gene causes a desmin-related myopathy. Nat Genet. 1998;20:92-5.

17. McClendon PL, Robbins J. Desmin-related cardiomyopathy: an unfolding story. Am J Physiol Heart Circ Physiol. 2011;301:H1220-8.

18. Saks V, Guzun R, Timohhina N, Tepp K, Varikmaa M, Monge C, et al. Structure function relationships in feedback regulation of energy fluxes in vivo in health and disease: mitochondrial interactosome. Biochim Biophys Act. 2010;1797: 678-97.

19. Dalakas MC, Park K-Y, Semino-Mora C, Lee HS, Sivakumar K, Goldfarb LG Desmin myopathy, a skeletal myopathy with cardiomyopathy caused by mutation of the desmin gene. New Eng J Med. 2000;342:770-80.

20. Wang X, Osinska H, Klevitsky R, Gerdes AM, Nieman M, Lorenz J, et al. Expression of R120G- $\alpha$-B-crystallin causes aberrant desmin and $\alpha \beta$-crystallin aggregation and cardiomyopathy in mice. Circ Res. 2001;89:84-91.

21. Milner DJ, Weitzer G, Tran D, Bradley A, Capetanaki Y. Disruption of muscle architecture and myocardial degeneration in mice lacking desmin. J Cell Biol. 1996; 134:1255-70.

22. Nakamura K, Kusano K, Nakamura Y, Kakishita M, Ohta K, Nagase S, et al. Carvedilol decreases elevated oxidative stress in human failing myocardium. Circulation. 2002; 105:2867-71.

23. Bennardini F, Wrzosek A, Chiesi M. $\alpha$-B-crystallin in cardiac tissue. Association with actin and desmin filaments. Circ Res. 1992;71:288-94.

24. Agnetti G, Halperin VL, Kirk JA, Chakir K, Guo Y, Lund L, et al. Desmin modifications associate with amyloid-like oligomers deposition in heart failure. Cardiovasc Res. 2014;102:24-34.

25. Yancey D, Guichard J, Ahmed MI, Zhou L, Murphy MP, Johnson MS, et al. Cardiomyocyte mitochondrial oxidative stress and cytoskeletal breakdown in the heart with a primary volume overload. Am J Physiol Heart Circ Physiol. 2015; 308:H651-63.

26. Di Somma S, Di Benedetto MP, Salvatore G, Agozzino L, Ferranti F, Esposito S, et al. Desmin-free cardiomyocytes and myocardial dysfunction in end stage heart failure. Eur J Heart Fail. 2004;6:389-98.

27. Di Somma S, Marotta M, Salvatore G, Cudemo G, Cuda G, De Vivo F, et al. Changes in myocardial cytoskeletal intermediate filaments and myocyte contractile dysfunction in dilated cardiomyopathy: an in vivo study in humans. Heart. 2000;84:659-67.

28. Oral H, Sivasubramanian N, Dyke DB, Mehta RH, Grossman PM, Briesmiester $\mathrm{K}$, et al. Myocardial proinflammatory cytokine expression and left ventricular remodeling in patients with chronic mitral regurgitation. Circulation. 2003;107:831-7.

29. Grossman PM, Linares OA, Supiano MA, Oral H, Mehta RH, Starling MR. Cardiac-specific norepinephrine mass transport and its relationship to left ventricular size and systolic performance. Am J Physiol Heart Circ Physiol. 2004;287: H878-88.

30. Zheng J, Yancey D, Ahmed MI, Wei CC, Powell PC, Shanmugam M, et al. Increased Sarcolipin expression and adrenergic drive in patients with chronic isolated mitral regurgitation. Circ Heart Fail. 2014;7:194-202.

31. Pappa Z, van der Veldenb J, Stienen GMM. Calpain-I induced alterations in the cytoskeletal structure and impaired mechanical properties of single myocytes of rat heart. Cardiovasc Res. 2000;45:981-93.

32. Goll DE, Thompson VF, Li H, Wei W, Cong J. The calpain system. Physiol Rev. 2003;83:731-801.

33. Pat B, Killingsworth C, Denney T, Zheng J, Powell P, Tillson M, et al. Dissociation between cardiomyocyte function and remodeling with beta-adrenergic receptor blockade in isolated canine mitral regurgitation. Am J Physiol Heart Circ Physiol. 2008;295:H2321-37.

34. Tsutsui H, Spinale FG, Nagatsu M, Schmid PG, Ishihara K, DeFreyte G, et al. Effects of chronic beta-adrenergic blockade on the left ventricular and cardiocyte abnormalities of chronic canine mitral regurgitation. J Clin Invest. 1994;93: 2639-48.

35. Kamlot A, Bellows SD, Simkhovich BZ, Hale SL, Aoki A, Kloner RA, et al. Is warm retrograde blood cardioplegia better than cold for myocardial protection? Ann Thorac Surg. 1997;63:98-104.

Key Words: heart failure, mitochondria, cardiomyocyte, mitral regurgitation 

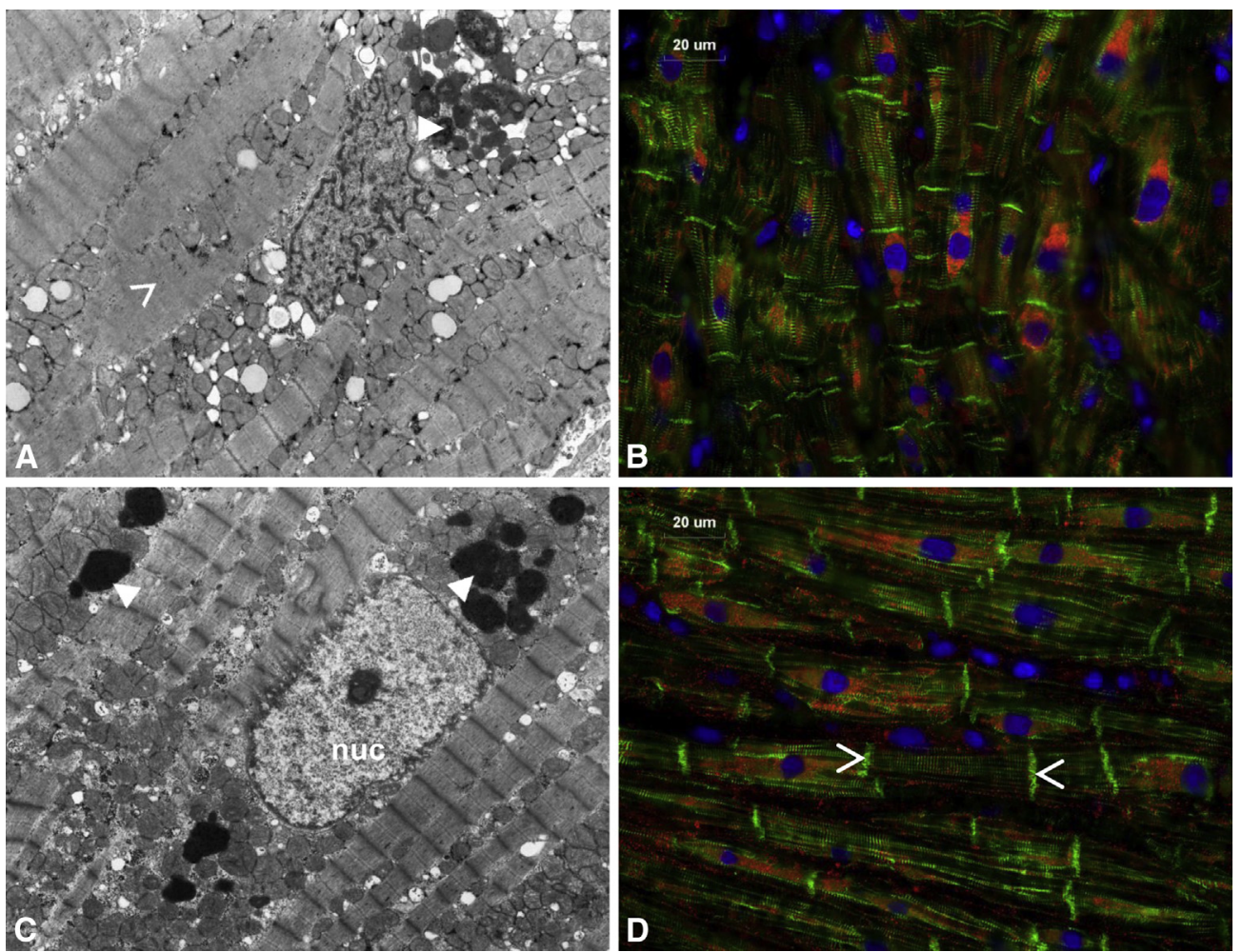

FIGURE E1. A and C, Transmission electron microscopy (TEM) (5000×) and B and D, Immunohistochemistry (IHC) for desmin (green) and $\alpha$, $\beta$-unsaturated aldehyde 4-hydoxynonenal (4-HNE) (red) for patients 3 (A and B) and 6 (C and D) in Table E2. There is extensive breakdown of desmin shown in the IHC images, as demonstrated in 1 sarcomere between 2 intercalated discs (open arrowheads) with a marked increase in 4-HNE, especially in the perinuclear area. TEM images demonstrate marked increase in electron-dense aggregates (closed arrowheads) that correspond to an increase in perinuclear 4-HNE staining and numerous lipid droplets, which are shown as clear round vacuoles. There is extensive disruption and loss of Z-disc (open arrowheads) and mitochondrial clustering and disarray throughout the cardiomyocyte. nuc, Nucleus.
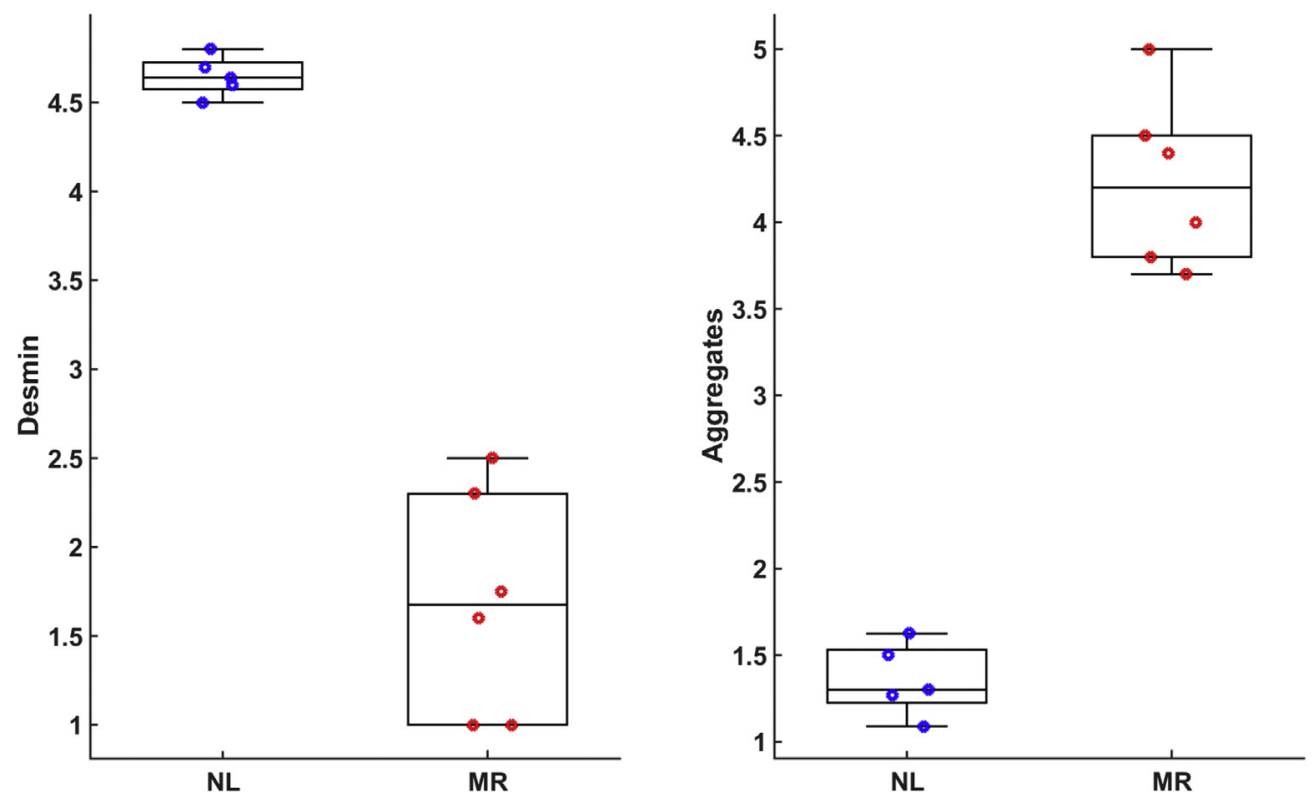

FIGURE E2. Box-and-whisker plots with the data points superimposed to show the quantitation of desmin loss and aggregate as percent of area of cardiomyocytes $(P=.0043$ vs normal $[N L]) . M R$, Mitral regurgitation. 
TABLE E1. Patient demographic characteristics

\begin{tabular}{|c|c|c|c|c|c|c|c|c|c|c|c|}
\hline Sex & Race & Age (y) & Medications & Hypertension & $\begin{array}{c}\text { NYHA } \\
\text { functional } \\
\text { class } \\
\end{array}$ & Date & $\begin{array}{c}\text { LVEDV } \\
(\mathrm{mL})\end{array}$ & $\begin{array}{c}\text { LVESV } \\
(\mathbf{m L})\end{array}$ & $\begin{array}{c}\text { LVEDD } \\
(\mathbf{c m})\end{array}$ & $\begin{array}{c}\text { LVESD } \\
(\mathrm{cm})\end{array}$ & $\begin{array}{c}\text { LVEF } \\
(\%) \\
\end{array}$ \\
\hline M & $\mathrm{W}$ & 58 & $\beta$-blocker, diuretic & Yes & 2 & 2005 & 152 & 43 & 5.44 & 3.38 & 72 \\
\hline M & $\mathrm{W}$ & 50 & None & No & 1 & 2005 & 240 & 100 & 7.08 & 5.29 & 58 \\
\hline M & $\mathrm{W}$ & 71 & ACEi, $\beta$-blocker & Yes & 2 & 2005 & 176 & 69 & 5.54 & 3.60 & 61 \\
\hline M & $\mathrm{W}$ & 62 & $\mathrm{ACEi}$ & No & 2 & 2005 & 183 & 72 & 5.47 & 4.18 & 61 \\
\hline $\mathrm{F}$ & $\mathrm{W}$ & 44 & None & No & 2 & 2005 & 159 & 64 & 6.17 & 3.67 & 60 \\
\hline $\mathrm{F}$ & & 48 & $\mathrm{ACEi}$, diuretic, $\beta$-blocker & Yes & 2 & 2006 & 165 & 60 & 6.30 & 4.42 & 64 \\
\hline M & W & 47 & $\mathrm{ACEi}, \beta$-blocker, statin & Yes & 2 & 2006 & 222 & 59 & 6.57 & 4.48 & 74 \\
\hline M & $\mathrm{W}$ & 33 & diuretic & No & 2 & 2006 & 263 & 52 & 6.65 & 3.90 & 80 \\
\hline M & $\mathrm{W}$ & 49 & None & No & 1 & 2006 & 164 & 63 & 6.01 & 4.08 & 62 \\
\hline M & $\mathrm{W}$ & 61 & None & No & 1 & 2007 & 217 & 77 & 6.60 & 4.61 & 65 \\
\hline M & W & 33 & None & No & 1 & 2007 & 233 & 67 & 6.43 & 4.27 & 71 \\
\hline $\mathrm{F}$ & $\mathrm{W}$ & 76 & ACEi & Yes & 2 & 2009 & 174 & 76 & 5.64 & 4.62 & 56 \\
\hline M & $\mathrm{W}$ & 80 & AT1RB & Yes & 2 & 2007 & 155 & 52 & 5.49 & 4.14 & 67 \\
\hline $\mathrm{F}$ & $\mathrm{W}$ & 56 & Diuretic & Yes & 2 & 2008 & 98 & 28 & 4.55 & 2.90 & 72 \\
\hline $\mathrm{F}$ & $\mathrm{W}$ & 62 & $\beta$-blocker & No & 3 & 2008 & 201 & 94 & 6.19 & 4.32 & 53 \\
\hline $\mathrm{F}$ & $\mathrm{W}$ & 55 & None & No & 2 & 2008 & 215 & 81 & 6.14 & 5.31 & 62 \\
\hline M & $\mathrm{W}$ & 52 & $\beta$-blocker, diuretic & No & 2 & 2008 & 306 & 115 & 7.11 & 4.83 & 62 \\
\hline M & $\mathrm{W}$ & 62 & $\begin{array}{l}\beta \text {-blocker, calcium entry } \\
\text { blocker }\end{array}$ & Yes & 1 & 2008 & 286 & 74 & 6.78 & 3.71 & 74 \\
\hline $\mathrm{F}$ & W & 64 & None & No & 2 & 2009 & 132 & 60 & 5.16 & 3.79 & 54 \\
\hline M & $\mathrm{W}$ & 58 & ACEi, $\beta$-blocker & Yes & 1 & 2009 & 197 & 42 & 5.77 & 4.26 & 79 \\
\hline $\mathrm{F}$ & $\mathrm{W}$ & 60 & Digoxin, diuretic, $\beta$-blocker & Yes & 3 & 2009 & 207 & 82 & 5.62 & 4.54 & 61 \\
\hline $\mathrm{F}$ & $\mathrm{W}$ & 67 & $\beta$-blocker, diuretic, statin & No & 2 & 2009 & 153 & 51 & 5.54 & 3.47 & 66 \\
\hline
\end{tabular}

NYHA, New York Heart Association; $L V E D V$, left ventricular end-diastolic volume; $L V E S V$, left ventricular end-systolic volume; $L V E D D$, left ventricular end-diastolic dimension; $L V E S D$, left ventricular end-systolic dimension; $L V E F$, left ventricular ejection fraction; $M$, male; $W$, white; $A C E i$, angiotensin-converting enzyme inhibitor; $F$, female; $A T 1 R B$, type 1 angiotensin II receptor blocker.

TABLE E2. Demographic and echocardiographic imaging parameters

\begin{tabular}{|c|c|c|c|c|c|c|c|c|c|c|}
\hline $\begin{array}{c}\text { Patient } \\
\text { identification } \\
\text { number } \\
\end{array}$ & Sex & Race & Age & Medications & Hypertension & $\begin{array}{c}\text { NYHA } \\
\text { functional class }\end{array}$ & Date & $\begin{array}{c}\text { LVEDD } \\
(\mathbf{m m})\end{array}$ & $\begin{array}{c}\text { LVESD } \\
(\mathbf{m m})\end{array}$ & $\begin{array}{c}\text { LVEF } \\
(\%)\end{array}$ \\
\hline 1 & $\mathrm{~F}$ & W & 60 & $\beta$-blocker & No & 2 & 2015 & 54 & 34 & $>55$ \\
\hline 2 & M & $\mathrm{W}$ & 81 & ACEi, $\beta$-blocker & Yes & 2 & 2015 & 46 & 34 & $>55$ \\
\hline 3 & M & $\mathrm{W}$ & 62 & None & No & 2 & 2015 & 53 & 32 & $>55$ \\
\hline 4 & $\mathrm{~F}$ & $\mathrm{~W}$ & 58 & Diuretic & No & 2 & 2016 & 49 & 31 & $>55$ \\
\hline 5 & M & $\mathrm{W}$ & 39 & $\beta$-blocker, statin & No & 2 & 2016 & 61 & 29 & $>55$ \\
\hline 6 & M & W & 50 & None & No & 2 & 2016 & 56 & 37 & $>55$ \\
\hline
\end{tabular}

NYHA, New York Heart Association; $L V E D D$, left ventricular end-diastolic dimension; $L V E S D$, left ventricular end-systolic dimension; $L V E F$, left ventricular ejection fraction; $F$, female; $W$, white; $M$, male; $A C E i$, angiotensin-converting enzyme inhibitor. 